\title{
Model Based On-line Energy Prediction System for Semi-Autonomous Mobile Robots
}

\author{
Ramviyas Parasuraman, Keith Kershaw \\ Engineering department \\ European Organization for Nuclear Research (CERN) \\ Geneva 1211, Switzerland \\ ramviyas.np@cern.ch, keith.kershaw@cern.ch
}

\author{
Prithvi Pagala, Manuel Ferre \\ Centre for Automation and Robotics UPM-CSIC \\ Univesidad Politècnica de Madrid (UPM) \\ Madrid 28006, Spain \\ ps.pagala@upm.es,m.ferre@upm.es
}

\begin{abstract}
Maximizing energy autonomy is a consistent challenge when deploying mobile robots in ionizing radiation or other hazardous environments. Having a reliable robot system is essential for successful execution of missions and to avoid manual recovery of the robots in environments that are harmful to human beings. For deployment of robots missions at short notice, the ability to know beforehand the energy required for performing the task is essential. This paper presents a on-line method for predicting energy requirements based on the pre-determined power models for a mobile robot. A small mobile robot, Khepera III is used for the experimental study and the results are promising with high prediction accuracy. The applications of the energy prediction models in energy optimization and simulations are also discussed along with examples of significant energy savings.
\end{abstract}

Keywords: Mobile robots, Energy management, Energy optimization, Energy prediction, Energy consumption models.

\section{INTRODUCTION}

Mobile robots are used in various applications such as remote inspection, survey and defence applications. The robot's energy consumption is dependent on the nature of tasks to be performed and the time to complete the assigned mission. In hostile, nuclear or disaster environments, the robots will be equipped with teleoperation capabilities and/or semiautonomous features to ease the operator overload. For these robotic applications, the main energy autonomy challenges to be considered are that the robot should be able to go over long distances and operate for relatively long periods.

Since robots deployed in hazardous environments usually carry a finite power source such as batteries (because of environmental constraints), it is critical to ensure before executing the mission that the robot has sufficient energy to complete the task by predicting the energy requirements before-hand. In addition, the robot should be able to take actions if the remaining energy in the robot is not enough to make a return trip to its charging station.

The robots need to manage their energy consumption to avoid running out of energy as this would mean personnel access to retrieve or recover the robot from the hazardous (e.g. radiation) area where it was deployed (For e.g. [1]). Also, the robot's energy autonomy should be optimized to provide more flexibility in possible interventions (in terms of distance travelled and mission time). Predicting the energy requirements of various components in a robot helps making decisions on optimizing the tasks and the mission to be accomplished with the available energy capacity. It is important to develop energyefficient designs considering multiple components together.

In this paper, we extend our work in [2] and propose an energy prediction modelling approach for a small scale mobile robot platform. This paper also discusses the applications of using energy prediction models in on-line energy monitoring, energy optimization, and simulations in autonomous or teleoperated robots. In the next section, we review the literature regarding energy management in mobile robots and in the "Methodology" section, the theoretical basis behind this work is discussed. Finally, we present the experimental set-up and discuss the results along with the energy model applications.

\section{RELATED WORK}

The significance of power management for long-term operation of autonomous robots is discussed in the work by Deshmukh et al. [3], with challenges in terms of battery technology, power estimation and auto recharging. In [4], a robot with an auto-recharging system is proposed with emphasis on the aspects improving the robustness (or reliability) of the system. A method for automatically recharging the batteries using the robot's built-in sensors to control docking with a recharging station was proposed in [5]. Much research has been conducted on mobile robot motion energy optimization through motion planning [6], [7] and path planning [8], [9] techniques. Models for locomotion power and dynamics have been widely studied. Wei Yu et al [10] emphasize that power models of motors are needed for the locomotion planning to complete time or energy constrained missions. Power models for skid steered wheeled [10], [11] and tracked vehicles [12] are proposed for various turning radius and surface conditions.

Some works analyse the energy consumption of different components in robots. Liu et al. [13] present an energy breakdown table of a Mars rover. Michaud et al. [14] estimate the energy consumption of a rover including the communication power. However, they do not build power models for each component. A study [15] indicates that sensing, computation and communication consume significant amounts of power compared to locomotion power. Therefore, management of all power consuming modules is important. In [16], power models were used to optimize the deployment of robots under energy and timing constraints. The MarXbot [17] can autoreplace its batteries with hot swapping capability. In [17], it is proposed that power models of various components allow the robot to estimate when the robot should exchange its battery. A 
nice behavioural model for finding the optimal time at which a robot (ot the mobile agent) should go back to recharge is presented in [18].

Hostile or scientific facilities (such as at CERN) generally are not designed to accommodate mobile teleoperated robots. It is complicated to navigate the mobile robot to the work site due to the compact spaces and unstructured sections. For e.g. special provisions were made for the TIM robot [19] to pass through the sector doors in the Large Hadron Collider at CERN. Because of such restrictions, auto-recharging techniques [3]-[5], [18] cannot be used for telerobotic applications in scientific facilities emitting ionizing radiations.

TABLE I: COMPARISON OF VARIOUS MOBILE ROBOTS MASS AND POWER CONSUMPTION

\begin{tabular}{c|c|c} 
Robot & Mass $(\mathrm{kg})$ & Maximum locomotion power (W) \\
Khepera III [This paper] & 0.69 & $0.8(28 \%)$ \\
MarXbot [17] & 1 & $3.5(33 \%)$ \\
Pioneer DX [15] & 9 & $10.6(34 \%)$ \\
Auriga- $\beta$ [12] & 286 & $3000(87 \%)$ \\
TIM [19] & 500 & $2000(92 \%)$
\end{tabular}

Table I gives a comparison of some mobile robot's mass and power consumption considering the maximum power for each component. It can be observed that as the robot becomes heavier; the locomotion power accounts for higher percentage of the total power because the locomotion power depends on the size and the mass of the robot while the computers and sensors are relatively independent of the robot's size and mass. It should be noted that, adding more batteries to the robot adds more mass thereby requiring more power for locomotion.

Analysis of previous studies suggest that there is no common approach for creating power and energy consumption models of various components irrespective of the type of mobile robot and hence a generic modular approach for building power models and predicting the energy consumption of a mobile robot is proposed. This work aims towards the use of robots for swarm robotic applications (e.g. Khepera III), radiation inspection applications (e.g. TIM) in hazardous environment and specifically towards ensuring successful completion of task from the energy perspective.

\section{Methodology}

\section{A. Teleoperated robotic system}

A general architecture of a teleoperated robot and the components used in the proposed energy management module (in dotted-line boxes) is shown in Fig 1. As ex-plained in [12], mobile robots usually have multiple components, such as motors, sensors, and microcontrollers, embedded computers and communication devices. Communication with the robot can be either wired or wireless. Embedded computers are used for high-level computation and micro-controllers for low-level controls. The remote control station (commanding the robot) usually consists of communication devices, computers, information or video display modules, input and control devices such as joystick, keyboard and touch panel.

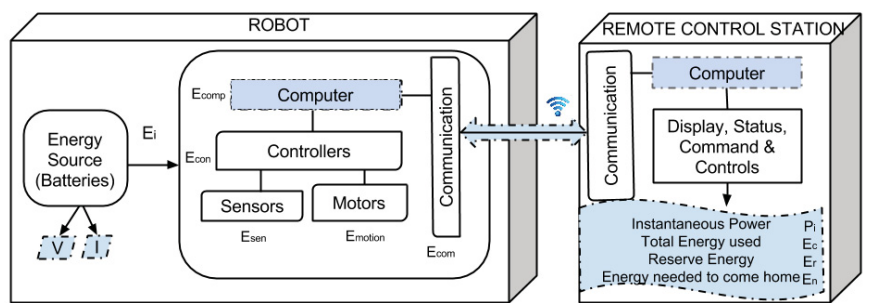

Fig. 1: General architecture of teleoperated robotic system highlighting the components used in the energy monitoring system

The time-stamped instantaneous battery voltage and total current used by the robot (instead of measuring current through each component) is used to build the power models. The energy needed by the robot to preform any task (including the on-line estimation of energy required to return to its base station) can be predicted with the help of these power consumption models. Most of the robots provide internal battery voltage information. Some robots are equipped with a battery charging system that provides both voltage and current information at run time.

\section{B. On-line energy prediction modelling system}

Power consumed by the robot depends on the number of active components; whereas, the energy consumption depends on the power consumption as well as the amount of time each component is active. A one-time process for generating the power models for various components can be developed by conducting some pre-determined series of operations on the robot (e.g. Fig. 2) either manually or programmed operations in the robot's embedded computer. When an additional component (hardware) is added to the robot, the robot's existing power model is updated. This feature makes the energy prediction algorithm modular and expandable for future modifications in the robot.

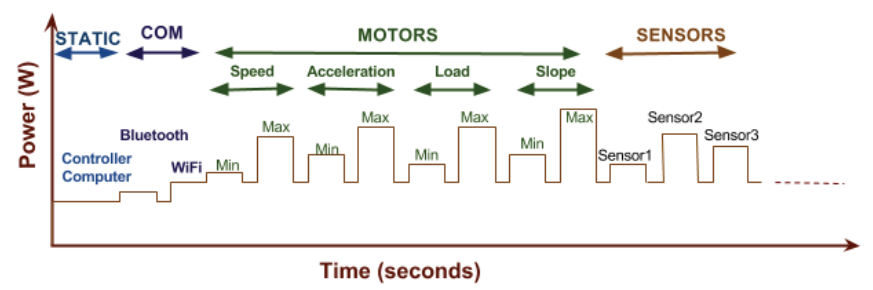

Fig. 2: Sequence of operations to determine power models of various components in a mobile robot

The instantaneous power consumption $\mathrm{Pi}$ of the mobile robot is the sum of power consumed by static and dynamic power consuming components. Components such as computer, controllers, and communication devices consume steady state power $P_{\text {static }}$ (without many fluctuations). Whereas components such as sensors and motors whose power consumption dynamically varies based on their usage are categorizes as dynamic components with power $P_{\text {dynamic }}$. The instantaneous power $P^{i}$ at time instant $i$ is given by: 


$$
P^{i}=\sum_{i=1}^{n_{1}} P_{\text {static }}^{i}+\sum_{i=1}^{n_{2}} P_{\text {dynamic }}^{i}, \text { where } n=n_{1}+n_{2}
$$

Here, $n_{1}$ is the number of static components, $n_{2}$ is the number of dynamic components and $n$ is the total number of components in the mobile robot. The energy consumed by a component $j$ is the summation of the power consumed by that component during the time $\left(t_{j}\right)$ the component is active.

$$
E_{c_{j}}=\sum_{i=1}^{t_{j}} P_{c_{j}}^{i}
$$

To obtain the total energy consumed by the robot $\left(E_{c}\right)$ in driving along a path of length $p$ for $t_{p}$ seconds, integrate all the $n$ components' energy consumption as follows:

$$
\begin{aligned}
E_{c} & =E_{\text {static }}+E_{\text {dynamic }} \\
& =\int_{0}^{t_{p}} \sum_{j=1}^{n}\left(G_{c_{j}}^{i} \cdot P_{c_{j}}^{i}\right) \mathrm{d} t
\end{aligned}
$$

Where $P_{c_{j}}^{i}$ is the maximum power consumed by a component $j$ at time instant $i$ and $G_{c_{j}}^{i}$ is the weight function applied to each component based on its type as follows:

$$
G_{c_{j}}^{i}= \begin{cases}1 & \text { when component } \mathrm{j} \text { is active and static } \\ \alpha & \text { when component } \mathrm{j} \text { is active and dynamic } \\ 0 & \text { when component } \mathrm{j} \text { is passive }\end{cases}
$$

Where, $\alpha \leq 1$ is a parameter depends on the duty cycle (if it's a motor) or the frequency $f$ (if it's a sensor) of the dynamic component that is active at the instant $i$. For static components, the maximum power consumed is approx. equal to the average power consumed by them. For instance, the static components such as computers and controllers are always active in a mobile robot. Whereas, the communication devices need not be necessarily active all the time but can be passive for some duration. The power consumed by a sensor operating at a frequency $f$ can be modelled using a linear equation involving two constants $s_{1}$ and $s_{2}$ as [15]:

$$
P_{\text {sensors }}=s_{1} * f+s_{2}
$$

The motion power depends on various parameters such as the linear $v$ and angular velocity $\omega$, linear and angular accelerations $a, \dot{\omega}$, mass of the robot $m$ and the slope of the surface $\alpha$.

$$
P_{\text {motion }}^{i}=f(v, \omega, a, \dot{\omega}, m, \alpha)
$$

Most of the mobile robots use multiple DC motors for loco-motion with wheels, tracks or modular arrangements. The power model for DC motor with velocity $v$ and acceleration $\dot{v}$ at time instant $i$ is given in [20] as follows:

$$
\begin{aligned}
P_{\text {motion }}^{i}=C_{1} \cdot a^{i^{2}}+C_{2} \cdot v^{i^{2}} & +C_{3} \cdot v^{i}+C_{4} \\
& +C_{5} \cdot a^{i}+C_{6} \cdot a^{i} \cdot v^{i}
\end{aligned}
$$

The parameters $C_{1}, C_{2}, \cdots, C_{6}$ depends on the motor and wheel characteristics such as torque constants, damping force, load inertias, and the nature of the travelling surface. When the robot travel a pre-defined path, the initial and final velocities are zero, and therefore the motion power model can be reduced to:

$$
\begin{array}{r}
P_{\text {motion }}^{i}=C_{1} \cdot a^{i^{2}}+C_{2} \cdot v^{i^{2}}+C_{3} \cdot v^{i}+C_{4} \\
E_{\text {motion }}=\int_{0}^{t_{p}}\left(C_{1} \cdot a^{i^{2}}+C_{2} \cdot v^{i^{2}}+C_{3} \cdot v^{i}+C_{4}\right) d t
\end{array}
$$

The total energy consumed by a robot is given below as a sum of energy consumed by computer, controller, sensors, motors and communication devices.

$$
E_{c}=E_{c o m p}+E_{c o n}+\sum_{j=1}^{n_{s}} E_{\text {sen }_{j}}+\sum_{j=1}^{n_{m}} E_{\text {motion }_{j}}+E_{c o m}
$$

Once the power model is generated, it can be used to calculate the on-line energy consumption of each component while the robot is performing some task or can be used to predict the energy requirements before the mission based on the nature of the tasks. The list of active components at any instant is determined by the operator (manual teleoperation) or by the program (autonomous). Different tasks or missions exhibit different energy consumption behaviour; nevertheless, the energy characterization will allow the operator to optimize the energy consumption.

Let $E_{a}$ be the total energy available in a mobile robot. Since we aim to predict the energy consumed during/before a mission, the dynamics of the total available energy capacity in a robot (that depends on the battery chemistry) is out of scope of this paper and we treat $E_{a}$ as constant. The reserve energy available at any instant is given by

$$
E_{r}^{i}=E_{a}-E_{c}^{i}
$$

Let us assume that the robot is active for $t_{n}$ seconds during a mission of travelling a path of length $d_{p}$ with an constant velocity $v_{p}$. The time taken by a robot to travel the distance $t_{p}=\frac{d_{p}}{v_{p}}$ (such that $t_{p} \leq t_{n}$ ) is the time during which the motors are active. The energy needed to execute this mission $E_{n}$ and the reserve energy $E_{r}$ after the execution of this mission will be:

$$
\begin{array}{r}
E_{n}=\left(P_{\text {static }} \cdot t_{n}+P_{\text {motion } @ u_{i}} \cdot t_{p}\right) \\
E_{r}=E_{a}-E_{n}
\end{array}
$$




\section{EXPERIMENTAL SETUP}

The Khepera III mobile robot $\left(127 \times 123 \times 70 \mathrm{~mm}^{3}\right.$, Fig 4), produced by the K-team Corporation, has an inbuilt smart battery monitoring system providing the current state of the battery (voltage, current, capacity remaining, and temperature) and hence this robot was selected to start with our experiments so that no additional hardware module (e.g. current sensor) was needed.

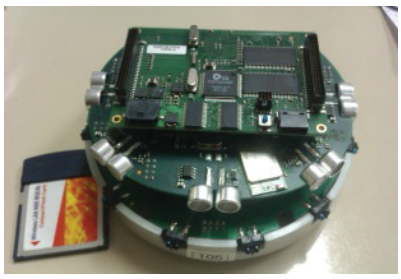

(a) Opened

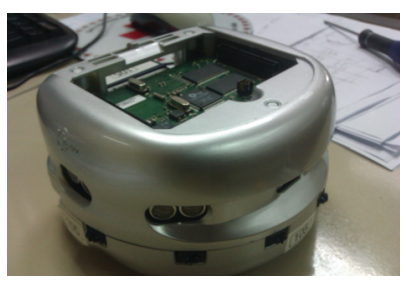

(b) Closed
Fig. 4: Khepera mobile robot

Khepera III powered by a rechargeable Li-polymer battery pack of $1400 \mathrm{mAh}$, has 2 DC brushed servo motors, 11 infrared sensors and 5 ultrasonic sensors. Korebot II, a minicomputer (extension module) that hosts Bluetooth and Wi-Fi communication modules is added to the Khepera III robot. The voltage and current values are recorded at a sampling frequency of $20 \mathrm{~Hz}$.

As soon as the robot is started, the power consumed by various components is recorded by activating or controlling the components one by one. Programs for deriving the power models from a series of operations (Fig. 4, right) and calculating the energy consumption of each component (by determining the duration for which each component was active) were written in MATLAB and tested with the robot's datasets created during the experiments. Initially the computer is turned on and hence $P_{\text {comp }}$ is calculated. Then, the controllers are activated and the power $P_{c o n}$ is noted. Each other component's power consumption is calculated by subtracting from the previous power value after the activation of the respective components.

Since all sensors data were needed during teleoperation to ensure safety of the robot and surroundings, all the sensing elements were active at maximum frequency throughout the experiments. Motors are the only dynamic components. Hence the static power component is the sum of sensing, controller and computer power. $P_{\text {static }}=P_{\text {sen }}+P_{\text {comp }}+P_{\text {con }}+P_{\text {com }}$ and $P_{\text {dynamic }}=P_{\text {motion }}$. The computer consumes an average $0.5 \mathrm{~W}$ power. Therefore, the sensing and control power is obtained by subtracting $0.5 \mathrm{~W}$ from the static power.

As the Khepera mobile robot is small in size and mass, the power model for locomotion is assumed (and is evident in the experiments) to be linear with respect to the average speed (of both motors) and the only cases considered were constant payload on a flat surface. However for bigger robots, this assumption does not hold true and hence the motion energy model (Equation 7 or 9) should be used instead. The acceleration effects are negligible when compared to the effect of velocity on power consumed by the motor [15]. Hence, the equation 9 can further be reduced adding the effect of payload mass $m_{p}$ as:

$$
P_{\text {motion }}^{i}=C_{v} \cdot v^{i}+C_{m_{p}} \cdot m_{p}+C_{I}
$$

Where, the parameter $C_{I}$ depends on the robot's mass $m_{r}$ and its inertia.

The power consumption of static components is observed to be almost constant (average values are used). The range of speed used in teleoperation experiments is $28 \mathrm{~mm} / \mathrm{s}-138 \mathrm{~mm} / \mathrm{s}$. With the measurement of motion power corresponding to the speed and payload of the robot (Fig 3a), a least square fit to the equation 14 can be applied to the measured data arriving at the following equation.

$$
P_{\text {motion }}^{i}=2.4 \cdot v^{i}+0.22 \cdot m_{p}+0.36
$$

The generated power model (Fig 3c) is used for energy prediction modelling system. It can be noted that the WiFi module consumes significantly higher power than other components.

To verify the accuracy of the energy prediction models, we conducted two different type of tasks with the robot travelling from point $\mathrm{A}$ to point $\mathrm{B}$ (Fig $5 \mathrm{a}$ ). For the experiments, the total time for completing the task $t_{n}=125 \mathrm{~s}$ and the time

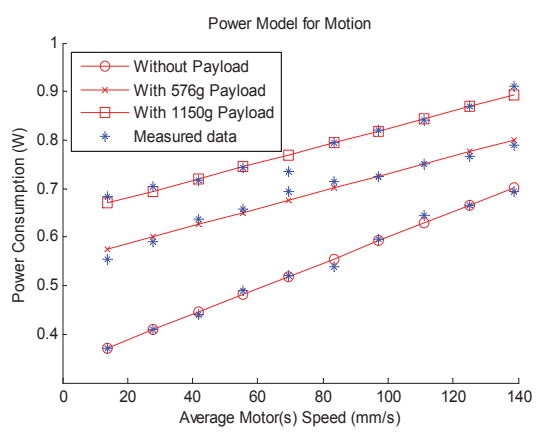

(a) Motion power with speed

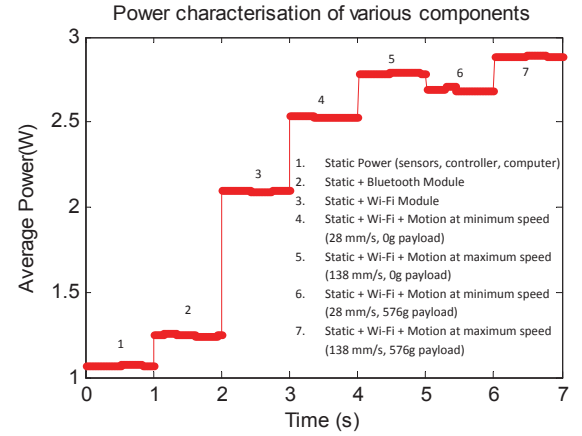

(b) Power characterization of the Khepera mobile robot

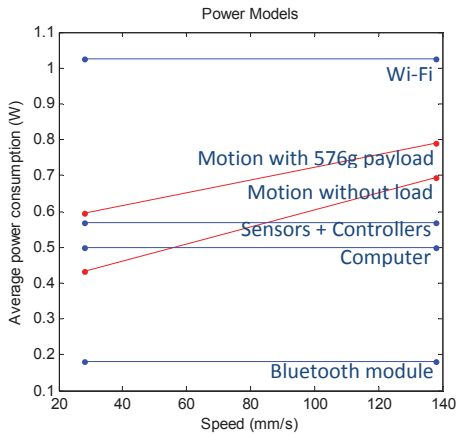

(c) Power models of various components

Fig. 3: Measured power models for Khepera mobile robot components 


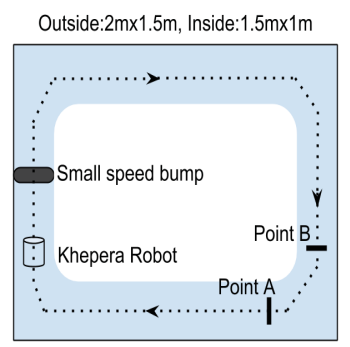

(a) Robot path during tasks
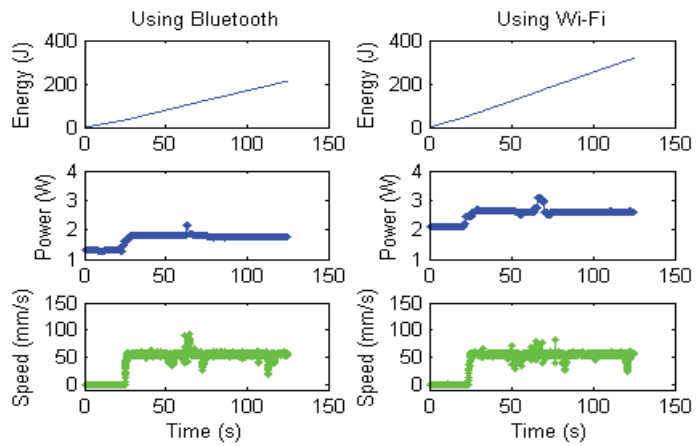

(b) Measured speed, power and energy during $\operatorname{task}^{A}$
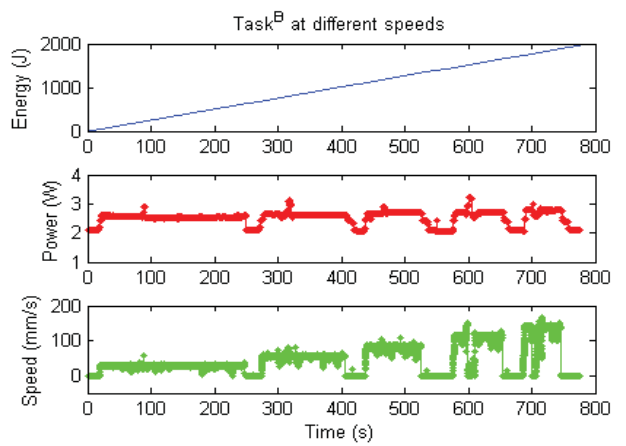

(c) Measured speed, power and energy during $\operatorname{task}^{B}$

Fig. 5: Experimental tasks performed and the measured values during the operation

for motion is $t_{p}=100 \mathrm{~s}$. The measurements sampling time $\mathrm{d} t=50 \mathrm{~ms}$.

The first task $\left(\operatorname{task}^{A}\right)$ is to run the robot with same velocity but with different static power consumers (i.e. the dynamic power consumption remains same in both case). In this scenario, the robot preformed the task twice, one using Wi-Fi module $\left(\operatorname{task}_{W i F i}^{A}\right)$ and again using Bluetooth module $\left(\operatorname{task}_{B t}^{A}\right)$. The robot moved at a constant velocity $v=56 \mathrm{~mm} / \mathrm{s}$. The measured speed, power and energy for $\operatorname{task} A$ (Fig $5 \mathrm{~b}$ ) show a minor power change at $70 \mathrm{~s}$ due to a small speed bump on the path. The second task $\left(\operatorname{tas}^{B}\right)$ is to circulate the robot in the robot arena (with point $\mathrm{A}$ as the origin) at different velocities. Figure $5 \mathrm{c}$ shows the measured speed, power and energy for task ${ }^{B}$.

The total energy drawn by the robot can be calculated by integrating the instantaneous power values (using voltage and current measurements)

$$
E_{r e f}=\sum_{i=0}^{t_{n}} V^{i} \cdot I^{i}
$$

This energy value (eqn. 16) is used as the ground truth reference value against which the predicted energy value using the energy models (equation 4) are compared. The predicted energy values $E_{\text {pred }}$ for $t a s k_{W i F i}^{A}$ and $t a s k_{B t}^{A}$ are computed using the derived power models in Fig $3 \mathrm{c}$ and the equations 4,15 and 13 .

\section{RESULTS AND DISCUSSIONS}

Figures 7 and 6 show the comparison of the reference energy values (computed with the measured data) and the predicted energy values (computed with the energy prediction models). The energy prediction error is calculated as:

$$
\operatorname{Error}(\%)=\frac{E_{\text {ref }}-E_{\text {pred }}}{E_{\text {ref }}} \cdot 100
$$

The mean error of the model-based energy calculation is $1.6 \%$ (with a range of $-2.8 \%$ to $+3.8 \%$. This proves that this method is highly accurate for application to energy prediction system to small scale robots.

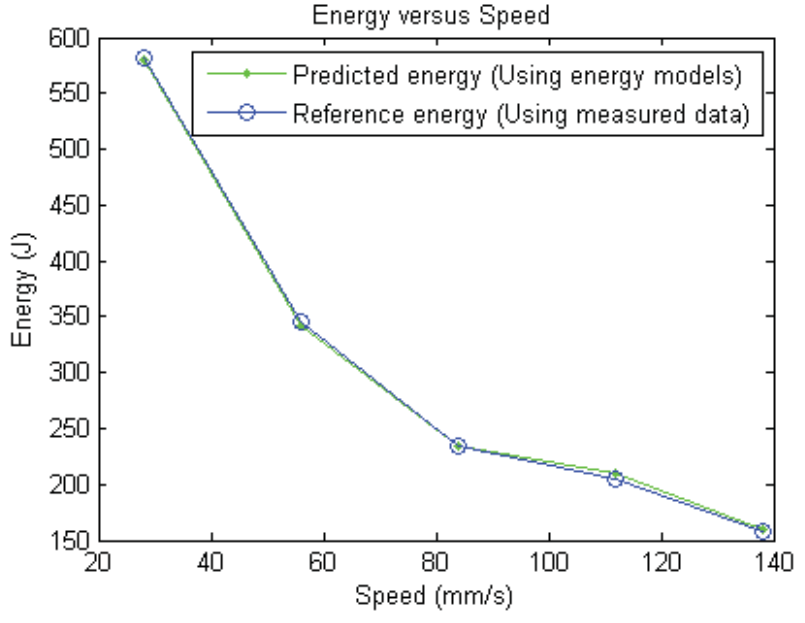

Fig. 6: Comparison of predicted (model-based) and measured (Reference) energy values with speed for $\operatorname{task}^{B}$

Considering the prediction error as $4 \%$ (eqn. 17), measurement error with the current and voltage transducers as $2 \%$ (from the transducer specifications), and a standard deviation from the average values as $4 \%$ (eqn.5), the energy prediction system should have a margin of at-least $10 \%$ for effective energy prediction and mission panning.

\section{A. Energy optimisation}

To travel from point A to point B, the energy saving made by operating the robot at higher speed $(138 \mathrm{~mm} / \mathrm{s})$ is $73 \%$ compared to operating the robot at $28 \mathrm{~mm} / \mathrm{s}$ (based on fig 6). It can be observed that around 33\% of energy can be saved by using the Bluetooth module instead of the WiFi module for communication. However, the Bluetooth standard has more constraints than WiFi (such as lesser distance range, lower data-rate capability). If the robot is operated at maximum speed and does not use any communication device, then the energy savings (computed using energy models) can be upto $83 \%$. Hence based on the system's needs and requirements, appropriate components have to be selected to minimize energy consumption. The energy savings depend on the type 

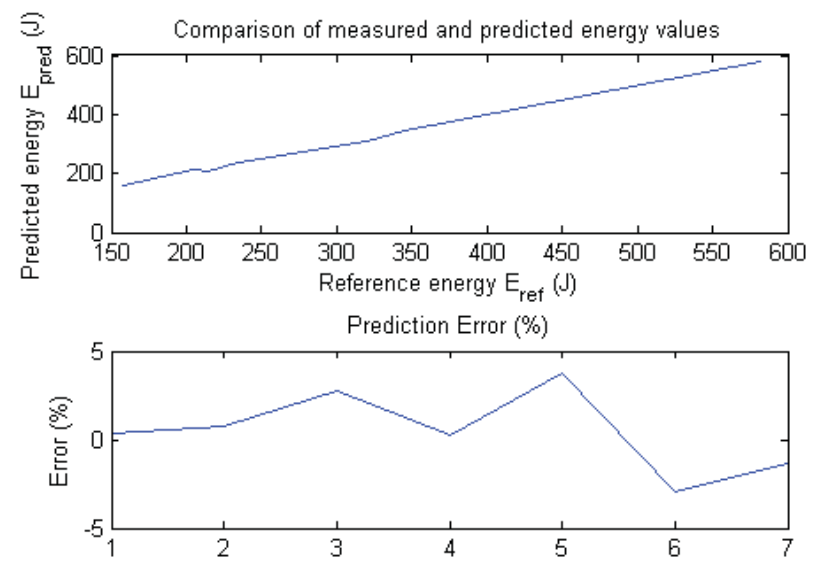

Fig. 7: Comparison of predicted (model-based) and measured (Reference) energy values showing the prediction error

of robot and the nature of the tasks.

This study is based only on a small robot and hence for bigger robots, other effects have to be considered such as battery's lifetime, proper cycling of charging and discharging the batteries and recovering energy through regenerative braking.

\section{B. Energy simulations}

Using the energy models, the tasks to be performed can be well planned to meet the energy demand of the robot. The simulated energy consumption models can be used to optimize and prepare robotic interventions by simulating the energy requirements. For instance, Player/Stage is a widely used simulator in the robotics research community to test robots' performance in a given environment. If the energy models are integrated into such simulators, it will be possible to simulate the energy consumption for specific tasks, situations and surroundings.

\section{CONCLUSIONS AND FURTHER WORK}

A generic approach for on-line energy prediction modelling system for a mobile robot is presented along with applications to energy optimization with an aim to provide increased safety and reliability for robots operating in hostile environments. The proposed method demonstrated high accuracy $(\geq 96 \%)$ during tests and can be also be used in saving energy by pre-predicting and pre-planning the robotic mission. A simple but effective and adaptive approach is followed so that this energy management feature can be used by many types of small-scale mobile robot (such as differential/skid steered, wheeled/tracked) as an additional module.

Future plans include integration of this energy prediction and optimisation module in a large sized robotic platform such as the TIM [19].

\section{ACKNOWLEDEGMENTS}

This research project has been supported by a Marie Curie Initial Training Network of the European Community's Sev- enth Framework Program under contract number PITN-GA2010-264336-PURESAFE.

\section{REFERENCES}

[1] K. Nagatani, S. Kiribayashi, Y. Okada, S. Tadokoro, T. Nishimura, T. Yoshida, E. Koyanagi, and Y. Hada, "Redesign of rescue mobile robot Quince," in 2011 IEEE International Symposium on Safety Security and Rescue Robotics. IEEE, 2011, pp. 13-18.

[2] R. Parasuraman, P. Pagala, K. Kershaw, and M. Ferre, "Energy Management Module for Mobile Robots in Hostile Environments," in Advances in Autonomous Robotics, ser. Lecture Notes in Computer Science, vol. 7429. Springer Berlin / Heidelberg, 2012, pp. 430-431.

[3] A. Deshmukh, P. Vargas, R. Aylett, and K. Brown, "Towards Socially Constrained Power Management for Long-Term Operation of Mobile Robots," in Towards Autonomous Robotic Systems (TAROS) Conference, UK, 2010.

[4] D. Austin and K. Kouzoubo, "Robust, Long Term Navigation of a Mobile Robot," in IARP/IEE-RAS Joint Workshop on Technical Challenges for Dependable Robots in Human Environments, 2002.

[5] S. Oh and A. Zelinsky, "Autonomous Battery Recharging for Indoor Mobile Robots," in Australian Conference on Robotics and Automation, 2000.

[6] Y. Mei, Y.-H. Lu, Y. Hu, and C. S. G. Lee, "Energy-efficient motion planning for mobile robots," in IEEE International Conference on Robotics and Automation, vol. 5, 2004, pp. 4344-4349 Vol.5.

[7] C. H. Kim and B. K. Kim, "Minimum-Energy Motion Planning for Differential-Driven Wheeled Mobile Robots," in Mobile Robots Motion Planning, New Challenges. Intechopen, 2008, pp. 193-226.

[8] H. Wei, B. Wang, Y. Wang, Z. Shao, and K. C. Chan, "Staying-alive path planning with energy optimization for mobile robots," Expert Systems with Applications, vol. 39, no. 3, pp. 3559 - 3571, 2012.

[9] Hui-zhong Zhuang and Shu-xin Du and Tie-jun Wu, "Energy-efficient motion planning for mobile robots," in International Conference on Machine Learning and Cybernetics, 2005.

[10] Wei Yu, Emmanuel Collins and Oscar Chuy, "Dynamic Modeling and Power Modeling of Robotic Skid-Steered Wheeled Vehicles," in Mobile Robots - Current Trends, D. Z. Gacovski, Ed. Intechopen, 2011.

[11] O. Chuy, J. Collins, E.G., W. Yu, and C. Ordonez, "Power modeling of a skid steered wheeled robotic ground vehicle," in IEEE International Conference on Robotics and Automation, 2009, pp. 4118-4123.

[12] J. Morales, J. Martinez, A. Mandow, A. Garcia-Cerezo, and S. Pedraza, "Power consumption modeling of skid-steer tracked mobile robots on rigid terrain," IEEE Transactions on Robotics, vol. 25, no. 5, pp. 1098$1108,2009$.

[13] J. Liu, P. Chou, N. Bagherzadeh, and F. Kurdahi, "Power-aware scheduling under timing constraints for mission-critical embedded systems," in Design Automation Conference, 2001, pp. 840-845.

[14] P. Lamon, "The solero rover," in 3D-Position Tracking and Control for All-Terrain Robots, ser. Springer Tracts in Advanced Robotics. Springer Berlin Heidelberg, 2008, vol. 43, pp. 7-19.

[15] Y. Mei, Y. Yung-Hsiang Lu Hu, and C. Lee, "A case study of mobile robot's energy consumption and conservation techniques," in 12th International Connference on Advanced Robotics, 2005, pp. 492-497.

[16] Y. Mei, Y.-H. Lu, Y. Hu, and C. S. G. Lee, "Deployment of mobile robots with energy and timing constraints," IEEE Transactions on Robotics, vol. 22, no. 3, pp. 507-522, 2006.

[17] M. Bonani, V. Longchamp, S. Magnenat, P. Retornaz, D. Burnier, G. Roulet, F. Vaussard, H. Bleuler, and F. Mondada, "The marxbot, a miniature mobile robot opening new perspectives for the collectiverobotic research," in International Conference on Intelligent Robots and Systems, IROS, Taiwan, 2010.

[18] J. Wawerla and R. T. Vaughan, "Optimal robot recharging strategies for time discounted labour," in Artificial Life XI: Proceedings of the Eleventh International Conference on the Simulation and Synthesis of Living Systems, 2008, pp. 670-677.

[19] K. Kershaw, C. Bertone, P. Bestmann, T. Feniet, D. Forkel-Wirth, J. L. Grenard, and N. Rousset, "Remotely Operated Train for Inspection and Measurement in CERN's LHC Tunnel," in Particle Accelerator Conference 2009, Vancouver, Canada, Apr 2009.

[20] P. Tokekar, N. Karnad, and V. Isler, "Energy-optimal velocity profiles for car-like robots," in International Conference on Robotics and Automation, 2011, pp. 1457-1462. 